Zugang zur internationalen Rechtserzeugung 


\section{Zugang zur Völkerrechtssetzung: Demokratische Legitimationsdefizite in der transnationalen Rechtsordnung am Beispiel der 2030 Agenda für Nachhaltige Entwicklung}

\section{Hannah Birkenkötter}

Abstract: Das Völkerrecht wirkt zunehmend in nationale Rechtsordnungen binein, allerdings vermittelt: Die Verschränkung nationaler und internationaler Rechtsordnungen ist heute durch die Abwesenheit von Linearität und die Gleichzeitigkeit verschiedener Prozesse von Rechtssetzung und-umsetzung geprägt. Der vorliegende Beitrag beschreibt diese Entwicklungen anhand der Agenda 2030 für Nachbaltige Entwicklung, die 2015 von der Generalversammlung der Vereinten Nationen verabschiedet wurde. Es wird zunächst die Verhandlungsgeschichte der Agenda 2030 und ibre institutionelle Verankerung dargestellt, und sodann Rekursivität, d.h. die Interaktion verschiedener Akteure auf unterschiedlichen Ebenen hinsichtlich des konkreten normativen Gehalts der Agenda, als zentrales Merkmal heutigen transnationalen rechtlichen Ordnens herausgearbeitet. Der Beitrag schließt mit Erwägungen dazu, warum diese Art des Völkerrechts vor neuartigen Fragen demokratischer Legitimation steht und wie diesen begegnet werden kann.

Das Völkerrecht lässt sich schon lange nicht mehr ausschließlich als zwischenstaatliches Recht, welches die nationale Rechtsordnung weitestgehend unberührt lässt, fassen. Bereits 1964 beobachtete Wolfgang Friedmann die Entwicklung des Völkerrechts von einem reinen Koordinationsrecht zwischen souveränen Staaten zu einem Kooperationsrecht der internationalen Gemeinschaft und stellte fest, dass das Völkerrecht zunehmend Gemeinschaftsbelange und bestimmte Themenbereiche positiv regelt. ${ }^{1}$ Die Zahl multilateraler völkerrechtlicher Verträge, die solche globalen Regelungen enthalten, hat seitdem noch einmal deutlich zugenommen; es gibt heute so gut wie keine grenzüberschreitenden Sachverhalte, die der völkerrechtlichen Regulierung entzogen wären. ${ }^{2}$ Viele Normen zeitgenössischer multilateraler Verträge erheben außerdem den Anspruch, Sachverhalte

* Ich danke Felix Fouchard, Isabelle Ley, sowie den Teilnehmer*innen der 61. Jungen Tagung Öffentliches Recht für hilfreiche Hinweise.

1 Friedmann, The Changing Structure of International Law, 1964, Kapitel 6.

2 Klabbers, Introduction to International Organizations Law, 3. Aufl. 2015, S. 1. 
auch innerhalb staatlicher Grenzen zu regulieren; sie heben den Schleier staatlicher Souveränität auf, durch den das Völkerrecht klassischerweise innerstaatliche und völkerrechtliche Sphären vermittelte. ${ }^{3}$ Das ist in Rechtsgebieten wie dem internationalen Menschenrechtsschutz, dem Umweltvöl-

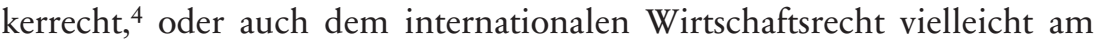
offensichtlichsten.

Der Anspruch international verhandelter Normen, in nationale Rechtsordnungen hineinzuwirken, ist nicht nur auf völkervertragsrechtliche oder völkergewohnheitsrechtliche Vorschriften begrenzt. Das zeitgenössische Völkerrecht produziert eine Vielzahl von Normen, die nicht ohne weiteres unter den tradierten Quellenkanon des Völkerrechts subsumiert werden können. ${ }^{5}$ Dazu zählt die Fülle von Empfehlungen internationaler Organisationen, darunter eine Reihe globaler Aktionsprogramme, und jüngst zunehmend Indikatoren, die ebenfalls international verhandelt werden und den Fortschritt der Umsetzung solcher Programme messen sollen. ${ }^{6}$ Solche transnationalen Regulierungsprozesse können begrifflich unterschiedlich gefasst werden, etwa als Global Administrative Law oder als informelles internationales Recht. ${ }^{7}$ Es ist ihnen aber gemein, dass die Grenzen zwischen staatlichem und internationalem Recht, zwischen privater und öffentlicher Regulierung, zwischen hard law und soft law, zunehmend verwischen.

Wenn die Verschränkung der internationalen mit nationalen Rechtsordnungen zunimmt, wenn immer mehr international verhandelte Normen den Anspruch erheben, das staatliche Recht zu formen, dann stellt sich mindestens in demokratisch verfassten Staaten die Frage nach der demokratischen Legitimation solcher Normen. Dabei wird das Demokratiedefizit des Völkerrechts sowohl über- als auch unterschätzt. Es wird überschätzt, wo davon ausgegangen wird, dass völkerrechtliche Normen einseitig, gleichsam „top-down“, in das nationale Recht hineinwirken. ${ }^{8}$ Viele, auch vertragsrechtliche, Normen sind vage formuliert und bieten dem na-

3 Besson, The Authority of International Law - Lifting the State Veil, Sydney Law Review 31 (2009), 343 (359).

4 Zur Genese dieses Teilrechtsgebiets jetzt umfassend und kritisch Boysen, Die postnationale Konstellation, 2020.

5 Goldmann, Internationale öffentliche Gewalt, 2015, S. 47-95.

6 Dazu Merry/Davis/Kingsbury, The Quiet Power of Indicators, 2015.

7 Kingsbury/Krisch/Stewart, The emergence of global administrative law, Law and Contemporary Problems 68 (2005), 15; Pauwelyn/Wessel/Wouters, Informal international lawmaking, 2012.

8 Ein Beispiel ist die 2018 formulierte Kritik am UN-Migrationspakt, siehe dazu Birkenkötter/Buszewski, Das Spiel hat gerade erst begonnen: Zur Kritik am Migrationspakt, VerfBlog, 2018/12/22, https://verfassungsblog.de/das-spiel-hat-gerade-erst-begonne 
tionalen, demokratischen Gesetzgeber einen Umsetzungs- und damit einen Gestaltungsspielraum. Gleichzeitig wird das Demokratieproblem aber dort unterschätzt, wo nur Normen des traditionellen völkerrechtlichen Quellenkanons, wie er paradigmatisch in Artikel 38 Abs. 1 IGH-Statut kodifiziert ist, überhaupt in den Blick genommen werden. Denn dann wird ein großer Teil international verhandelter Normen schlicht ignoriert, und zwar auch solche Normen, die durchaus einen Geltungsanspruch innerhalb nationaler Rechtsordnungen erheben - wenngleich dieser Geltungsanspruch stets ein vermittelter ist, wie im Folgenden noch gezeigt werden soll.

Der vorliegende Beitrag verfolgt vor diesem Hintergrund zwei Ziele: Erstens soll am Beispiel der Agenda 2030 für Nachhaltige Entwicklung9 gezeigt werden, wie internationale Normen heute einen Anspruch auf Einwirkung in nationale Rechtsordnungen erheben, gleichzeitig aber in hohem Maße prozesshaft sind und eigentlich nur aus ihrem weiteren Verhandlungskontext heraus verstanden werden können. Zweitens wird dann, ebenfalls am Beispiel der Agenda 2030, der Versuch einer begrifflichen Erfassung unternommen und aufgezeigt, warum diese Art des zeitgenössischen Völkerrechts vor neuartigen Fragen demokratischer Legitimation steht.

Dazu geht der Beitrag wie folgt vor: In einem ersten Schritt wird die Agenda 2030 und ihr Verhandlungsprozess näher vorgestellt. Sie steht beispielhaft für die Art von Agenden, die heute im Rahmen der Vereinten Nationen verhandelt werden und die in ihrem jeweiligen institutionellen Kontext zu sehen sind. Die Agenda 2030 kam nicht "aus dem Nichts“, sondern baute auf einem jahrzehntelangen, institutionalisierten Prozess auf. In einem zweiten Schritt wird dann der Begriff der transnationalen Rechtsordnungen vorgestellt und mit neueren Theorien transnationalen Regierens verknüpft. Hierdurch wird der Blick auf die Bedeutung von Teilhabe an internationalen Normverhandlungsprozessen geschärft. Wie Teilhabe in der Praxis aussieht, wird in diesem zweiten Abschnitt am Beispiel der Agenda 2030 und der Beteiligung von Frauen an ihrer Aushandlung gezeigt. Der Beitrag schließt in einem dritten Teil mit Erwägungen zur demokratischen Legitimation der Agenda 2030 und strukturell ähnlicher transnationaler Rechtsordnungen.

n-zur-kritik-am-migrationspakt/, DOI: 10.17176/20190211-224230-0 (letzter Aufruf am 2. April 2021).

9 UN Dok. A/Res/70/1. 


\section{Prozesshaftes Völkerrecht: Die Agenda 2030 für Nachhaltige Entwicklung}

2015 von der Generalversammlung der Vereinten Nationen verabschiedet, verspricht die Agenda 2030 für Nachhaltige Entwicklung nicht weniger als eine "Transformation der Welt" und ist entschlossen, „friedliche, gerechte und inklusive Gesellschaften aufzubauen“. ${ }^{10}$ Die Agenda 2030 ist seitdem die Leitschnur für das System der Vereinten Nationen mit seinen zahlreichen Unterorganisationen, Programmen und Fonds; das Jahrzehnt 2020-2030 wurde von Generalsekretär António Guterres zur „Aktionsdekade für Nachhaltige Entwicklung " ausgerufen. ${ }^{11}$ Zahlreiche VN-Unterorganisationen, allen voran das VN-Entwicklungsprogramm (UNDP), nutzen die Agenda 2030 als normative Zielvorgabe für konkrete Projekte vor Ort. So soll etwa in Angola die informelle Wirtschaft im Rahmen einer Reform sozialer Sicherungssysteme stärker in den Blick genommen werden, ${ }^{12}$ oder der Massentourismus in der Dominikanischen Republik umweltverträglicher und nachhaltiger gestaltet werden - und zwar explizit in Umsetzung der Agenda $2030 .{ }^{13}$ Aber auch private Initiativen in Industrieländern sind an der Agenda 2030 ausgerichtet: Der Verein Bluepingu e.V. etwa möchte in Nürnberg und Fürth die Agenda 2030 lokal umsetzen und bietet dazu u.a. Beratungen zu privaten Solaranlagen, ein Gemeinschaftsgartenprojekt oder einen Mietdienst für Lastenfahrräder an. ${ }^{14}$ Dies sind nur einzelne Beispiele für zehntausende Initiativen weltweit, die sich an der Agenda 2030 orientieren. ${ }^{15}$ In Deutschland hat die Agenda 2030 unter anderem auch zu

10 A/Res/70/1, Präambel und Ziff. 3.

11 Guterres, Remarks to High-Level Political Forum on Sustainable Development, 24. September 2019, https:/www.un.org/sg/en/content/sg/speeches/2019-09-24/re marks-high-level-political-sustainable-development-forum (letzter Aufruf am 28. April 2021).

12 UNDP Innovation, https://medium.com/@undp.innovation/understanding-and-e ngaging-with-the-complexity-of-informal-urban-markets-in-angola-631954216541 (letzter Aufruf am 28. April 2021).

13 UNDP Innovation, https://medium.com/@undp.innovation/transforming-the-tou rism-sector-in-the-dominican-republic-from-mass-market-to-a-resilient-system-fafo 72234c11 (letzter Aufruf am 28. April 2021).

14 Bluepingu, https://sdgs-go-local.bluepingu.de/ (letzter Aufruf am 28. April 2021).

15 Das letzte sog. „SDG Global Festival for Action“ der vom Generalsekretär der Vereinten Nationen ins Leben gerufenene SDG Action Campaign brachte virtuell knapp 25.000 Menschen zusammen, die an der Umsetzung der SDGs mitwirken: https:/globalfestivalofaction.org/about/ (letzter Aufruf am 28. April 2021). 
einer Debatte über die Frage geführt, ob Nachhaltigkeit ins Grundgesetz aufgenommen werden sollte. ${ }^{16}$

Das Herzstück der Agenda 2030 bilden 17 Ziele für Nachhaltige Entwicklung, die nochmals in 169 Unterziele gegliedert sind. Ob die Ziele erreicht sind, wird anhand von Indikatoren gemessen, die von der Statistischen Kommission der Vereinten Nationen entwickelt und in einer separaten Resolution der Generalversammlung zwei Jahre nach der Agenda 2030 verabschiedet wurden. ${ }^{17}$

\section{Ziele für nachhaltige Entwicklung (aus A/Res/70/1, S. 15) \\ Ziel 1: Armut in allen ihren Formen und überall beenden}

Ziel 2: Den Hunger beenden, Ernährungssicherheit und eine bessere Ernährung erreichen und eine nachhaltige Landwirtschaft fördern

Ziel 3: Ein gesundes Leben für alle Menschen jeden Alters gewährleisten und ihr Wohlergehen fördern

Ziel 4: Inklusive, gleichberechtigte und hochwertige Bildung gewährleisten und Möglichkeiten lebenslangen Lernens für alle fördern

Ziel 5: Geschlechtergleichstellung erreichen und alle Frauen und Mädchen zur Selbstbestimmung befähigen

Ziel 6: Verfügbarkeit und nachhaltige Bewirtschaftung von Wasser und Sanitärversorgung für alle gewährleisten

Ziel 7: Zugang zu bezahlbarer, verlässlicher, nachhaltiger und moderner Energie für alle sichern

Ziel 8: Dauerhaftes, breitenwirksames und nachhaltiges Wirtschaftswachstum, produktive Vollbeschäftigung und menschenwürdige Arbeit für alle fördern

Ziel 9: Eine widerstandsfähige Infrastruktur aufbauen, breitenwirksame und nachhaltige Industrialisierung fördern und Innovationen unterstützen

Ziel 10: Ungleichheit in und zwischen Ländern verringern

Ziel 11: Städte und Siedlungen inklusiv, sicher, widerstandsfähig und nachhaltig gestalten

Ziel 12: Nachhaltige Konsum- und Produktionsmuster sicherstellen

Ziel 13: Umgehend Maßnahmen zur Bekämpfung des Klimawandels und seiner Auswirkungen ergreifen*

Ziel 14: Ozeane, Meere und Meeresressourcen im Sinne nachhaltiger Entwicklung erhalten und nachhaltig nutzen

Ziel 15: Landökosysteme schützen, wiederherstellen und ihre nachhaltige Nutzung fördern,

Wälder nachhaltig bewirtschaften, Wüstenbildung bekämpfen, Bodendegradation beenden und umkehren und dem Verlust der biologischen Vielfalt ein Ende setzen

Ziel 16: Friedliche und inklusive Gesellschaften für eine nachhaltige Entwicklung fördern, allen Menschen Zugang zur Justiz ermöglichen und leistungsfähige, rechenschaftspflichtige und inklusive Institutionen auf allen Ebenen aufbauen

Ziel 17: Umsetzungsmittel stärken und die Globale Partnerschaft für nachhaltige Entwicklung mit neuem Leben erfüllen

"In Anerkennung dessen, dass das Rahmenübereinkommen der Vereinten Nationen über Klimaänderungen das zentrale internationale zwischenstaatliche Forum für Verhandlungen über die globale Antwort auf den Klimawandel ist.

16 Rat für Nachhaltige Entwicklung, https:/www.nachhaltigkeitsrat.de/aktuelles/gr undgesetz-for-future-nach-70-jahren-muss-die-verfassung-enkelfahiger-werden/ (letzter Aufruf am 28. April 2021).

17 UN Dok. A/Res/71/313. 
Bereits die Tatsache, dass Indikatoren und Ziele in zwei unterschiedlichen Dokumenten verabschiedet wurden, deutet an, dass die Agenda 2030 als Ergebnisdokument zwar für sich genommen gelesen und interpretiert werden kann, dass sich aber ihr eigentlicher Wert erst dann recht erschließt, wenn der Kontext und die Verhandlungsgeschichte in den Blick genommen werden. Die Agenda durchlief einen mehrjährigen, in vielfacher Hinsicht innovativen Verhandlungsprozess und brachte die bis dato im System der Vereinten Nationen getrennten Bereiche der Nachhaltigkeitspolitik und der Fortschreibung der Millenniumsentwicklungsziele zusammen. Diese Prozesshaftigkeit ist für heutige globale Agenden ebenso kennzeichnend wie die Vielzahl von Akteuren, die an ihrer Entstehung beteiligt sind. Beides soll im Folgenden überblicksartig skizziert werden, bevor in einem dritten Schritt die wichtigsten Inhalte der Agenda vorgestellt werden. ${ }^{18}$

\section{Die Agenda 2030 als Zusammenführung der Millenniumsentwicklungsziele und der Nachhaltigen Entwicklung}

Nachhaltigkeit ist seit Anfang der 1990er Jahre begrifflich in den Vereinten Nationen verankert. Der Begriff der Nachhaltigen Entwicklung wurde erstmalig im sog. Brundtland-Bericht als „Entwicklung, die die Bedürfnisse der Gegenwart befriedigt, ohne die Fähigkeit künftiger Generationen, ihre eigenen Bedürfnisse zu befriedigen, zu kompromittieren" definiert. ${ }^{19}$ Auf der Grundlage dieses Berichts bekannte sich die Staatengemeinschaft 1992 in Rio de Janeiro in der Rio-Erklärung zu 27 Prinzipien der nachhaltigen Entwicklung und verabschiedete einen mehrere hundert Seiten umfassenden Aktionsplan, die Agenda 21. ${ }^{20} \mathrm{Um}$ nachhaltige Entwicklung auch institutionell im System der Vereinten Nationen zu verankern, wurde eine Kommission für Nachhaltige Entwicklung eingerichtet, ${ }^{21}$ die mit dem Startschuss der Verhandlungen zur Agenda 2030 durch das Hochrangige Politische Forum abgelöst wurde. ${ }^{22}$

18 Zur Verhandlungsgeschichte der Agenda 2030 und der Nachhaltigkeitsziele Dodds/Donoghue/Leiva Roesch, Negotiating the Sustainable Development Goals, 2017.

19 Bericht der Kommission über Umwelt und Entwicklung, UN Dok. A/42/427, Annex, Kapitel 2, Ziff. 1.

20 UN Dok. A/CONF.151/26/REV.1(VOL.I).

21 Als Unterorgan des Wirtschafts- und Sozialrats, vgl. UN Dok. E/Res/1993/207.

22 UN Dok. A/Res/66/288, Ziff. 84. 
Die Millenniumsentwicklungsziele wiederum wurden in ihrer endgültigen Form - acht Ziele, die bis 2015 erreicht werden sollten - gar nicht zwischenstaatlich verhandelt, sondern durch eine vom VN-Generalsekretär einberufene Arbeitsgruppe auf der Grundlage der Millenniums-Erklärung der Generalversammlung aus dem Jahr 2000 entwickelt. ${ }^{23}$ Die Millenniums-Erklärung ist ihrerseits eine Zusammenführung und Höhepunkt verschiedener Entwicklungsagenden der 1990er Jahre, enthielt aber nur wenige und zudem schlecht operationalisierbare Ziele. ${ }^{24}$ Während das Ziel 7 der Millenniumsziele nachhaltige Entwicklung explizit einbezog, entstanden die Millenniumsentwicklungsziele institutionell unabhängig vom eben beschriebenen Rio-Prozess. Nicht nur, aber auch in diesem Politikbereich hatten die Millenniumsentwicklungsziele damit den unbeabsichtigten Nebeneffekt, bestehende institutionelle Prozesse zu unterbrechen und zu schwächen. ${ }^{25}$

Der Grundstein für Nachhaltige Entwicklungsziele wurde 2012 im Ergebnisdokument des Rio+20-Gipfels gelegt, einem Nachfolgegipfel des Rio-Gipfels von 1992. Auf dem Rio+20-Gipfel sprach sich die Staatengemeinschaft für die Erarbeitung messbarer Ziele für Nachhaltige Entwicklung auf der Grundlage der Agenda 21 und weiterer Dokumente im Nachgang zum ursprünglichen Rio-Gipfel aus. Zugleich wurde betont, solche Ziele müssten mit den Nachfolgezielen der Millenniumsentwicklungsziele integriert werden. ${ }^{26}$ Es entstand die Idee, beide Prozesse auch institutionell zusammenzuführen. Einen weiteren Schritt zur Integration der Nachhaltigen Entwicklung und der Post-2015-Agenda leistete der damalige Generalsekretär, Ban Ki-moon, indem er ein Hochrangiges Panel im Rahmen des Post-2015-Prozesses beauftragte, auch die Ergebnisse des Rio+20-Gipfels mit einzubeziehen. ${ }^{27}$ Dies sind die Grundsteine des Prozesses, der letztlich in die Agenda 2030 mündete und im folgenden Abschnitt beschrieben wird.

23 Vgl. die Ziele in UN Dok. A/56/326, Anhang.

24 Fukuda-Parr, Millennium Development Goals, 2017, S. 15.

25 Dazu Fukuda-Parr (mit Yamin und Greenstein), Millennium Development Goals, 2017, S. 147.

26 UN Dok. A/Res/66/288, Ziff. 245-251, hier Ziff. 246.

27 Terms of Reference for the High-level Panel of Eminent Persons on the Post-2015 Development Agenda, UN Dok. A/67/890, Anhang, S. 65. Vgl. auch Dodds/Donoghue/Leiva Roesch, Negotiating the Sustainable Development Goals (Fn. 18), S. 25. 
2. Ein neuartiger Verhandlungsprozess: Vom Hochrangigen Panel über die Offene Arbeitsgruppe zu zwischenstaatlichen Verhandlungen

Die Verhandlungen der Agenda 2030 erstreckten sich über mehrere Jahre und enthielten mehrere Schritte, durch die versucht wurde, so viele Akteure wie möglich in die Ausarbeitung der Agenda einzubeziehen. Das bereits erwähnte Hochrangige Panel legte seinen Abschlussbericht mit 12 ModellZielen im Mai 2013 vor. ${ }^{28}$ Von 2013 bis 2014 wurden dann die 17 Ziele und 169 Unterziele im Rahmen einer Offenen Arbeitsgruppe verhandelt. ${ }^{29}$ Dazu kamen eine Reihe von nationalen Konsultationen, die auch durch die VN-Präsenzen des VN-Entwicklungsprogramms vor Ort unterstützt wurden, sowie eine Online-Umfrage unter dem Titel „My World“, an der rund sieben Millionen Menschen teilnahmen. ${ }^{30}$ Dass der Verhandlungsprozess zur Agenda 2030 sowohl von Seiten des Generalsekretariats als auch von Seiten der Mitgliedstaaten mit größtmöglicher Transparenz und Offenheit für verschiedene Interessengruppen gestaltet wurde, ist auch auf Kritik an den Millenniumsentwicklungszielen zurückzuführen, die aus einem rein bürokratischen Prozess hervorgegangen waren. ${ }^{31}$

Die Offene Arbeitsgruppe sollte ursprünglich mit 30 Mitgliedstaaten aus den verschiedenen Weltregionen besetzt werden. Da sich die Staaten aber nicht einigen konnten, wer die jeweilige Region repräsentieren sollte, wurde jeder der 30 Sitze durch sogenannte „Duos“ und „Troikas“, also Staatenvertreter*innen von zwei bis drei Ländern, gemeinsam besetzt. ${ }^{32}$ Darüber hinaus spielten nichtstaatliche Akteure eine gewichtige Rolle. Auf dem Rio-Gipfel 1992 hatte die Staatengemeinschaft neun gesellschaftliche Hauptgruppen identifiziert, von denen die Umsetzung der Agenda 21 abhängen sollte: die Privatwirtschaft, Kinder und Jugend, die Landwirtschaft, indigene Bevölkerungsgruppen, Lokalregierungen, Nichtregierungsorganisationen, Forschung und Technik, Frauen sowie Arbeiter*innen/Gewerkschaften. ${ }^{33}$ Seitdem sind noch vier weitere Gruppen hinzugetreten: Menschen mit Behinderung, Freiwillige, ältere Menschen, sowie

28 UN Dok. A/67/890, Anhang.

29 Diese war durch das Ergebnisdokument des Rio+20-Gipfels eingesetzt worden, vgl. UN Dok. A/Res/66/288, Ziff. 248.

30 Fukuda-Parr, Sustainable Development Goals, in: Weiss/Daws (Hrsg.), Oxford Handbook on the United Nations, 2. Auflage, 2018, S. 769.

31 Fukuda-Parr, Sustainable Development Goals (Fn. 30), S. 769.

32 Dodds/Donoghue/Leiva Roesch, Negotiating the Sustainable Development Goals (Fn. 11), S. 31-32.

33 Vgl. Agenda 21, UN Dok. A/CONF.121/56 (Vol. III), Abschnitt III. 
das Bildungswesen und die Wissenschaft. ${ }^{34}$ Der Einfluss dieser Gruppen auf die Ausgestaltung der Agenda 2030 ist nicht zu unterschätzen. ${ }^{35}$ Dass die Vertreter*innen dieser Hauptgruppen, im VN-System bekannt als „Major Groups and other Stakeholders", aus dem Rio-Prozess für die Verhandlungen der Post-2015-Agenda als relevante Akteure angesehen wurden, zeigt, dass der Verhandlungsprozess der Agenda 2030 trotz der neuartigen Offenen Arbeitsgruppe in vergangenen Prozessen verhaftet geblieben ist.

An die Verhandlungsphase der Offenen Arbeitsgruppe schloss sich ab Oktober 2014 eine Phase zwischenstaatlicher Verhandlungen in der Generalversammlung an, wobei zunächst unklar war, ob die 17 Ziele und 169 Unterziele in dieser Phase - nicht zuletzt vor dem Hintergrund eines zusammenfassenden Berichts des Generalsekretärs, der noch einmal die aus seiner Sicht wichtigsten Aspekte einer Post-2015-Agenda hervorhob weiterhin verhandelbar sein sollten. ${ }^{36}$ Hier setzten sich die G77 und China durch; die zwischenstaatlichen Verhandlungen öffneten das Paket der Ziele und Unterziele nicht erneut, sondern konzentrierten sich stattdessen auf eine politische Erklärung, Indikatoren und den Folgeprozess sowie Mechanismen zur Überwachung der Umsetzung. ${ }^{37}$ Dabei ist zu erwähnen, dass der gerade aus der Perspektive der G77-Staaten überragend wichtige Aspekt der Finanzierung für Entwicklung (Financing for Development, FfD) nicht Teil des Agenda 2030-Dokuments ist. Stattdessen wurde diese Thematik in einem separaten Prozess zur Finanzierung von Entwicklung verhandelt, der in die Dritte Konferenz zur Entwicklungsfinanzierung und die dort verabschiedete Aktionsagenda von Addis Abeba für Nachhaltige Entwicklungsfinanzierung mündete. ${ }^{38}$

34 Vereinte Nationen, https://sustainabledevelopment.un.org/mgos (letzter Aufruf am 2. April 2021).

35 Dodds/Donoghue/Leiva Roesch, Negotiating the Sustainable Development Goals (Fn. 18), S. 62.

36 Synthesis-Bericht des Generalsekretärs der Vereinten Nationen in UN Dok. A/68/202.

37 Dodds/Donoghue/Leiva Roesch, Negotiating the Sustainable Development Goals (Fn. 18), S. 70-90, wobei die Indikatoren erst zwei Jahre später verabschiedet wurden, vgl. supra bei Fn. 17.

38 Befürwortet durch die Generalversammlung der Vereinten Nationen, UN Dok. A/Res/69/313, Anhang. 
3. „Niemanden zurücklassen“: Kerngehalte der Agenda 2030 für Nachhaltige Entwicklung und ihre völkerrechtliche Relevanz

Die Agenda 2030 für Nachhaltige Entwicklung, die in der Form einer Generalversammlungsresolution verabschiedet wurde, ist also das Ergebnis eines mehrjährigen Verhandlungsprozesses und enthält verschiedene Teile: die Politische Erklärung, die 17 Ziele und 169 Unterziele, und schließlich einen Teil zur Umsetzung und Nachverfolgung. ${ }^{39}$ Sie sollte außerdem gemeinsam mit der Aktionsagenda von Addis Abeba und den Globalen Indikatoren gelesen werden. ${ }^{40}$ Hier können nicht alle Inhalte der Agenda 2030 umfassend wiedergegeben werden. Es sei aber kurz auf ihre wichtigsten Leitprinzipien und Inhalte hingewiesen, um darzustellen, warum die Agenda völkerrechtlich von hoher Relevanz ist.

Die Politische Erklärung benennt gleich in der Präambel fünf Bereiche, unter die sich die Ziele subsumieren lassen sollen: Menschen, Planet, Wohlstand, Frieden, Partnerschaft, auch bekannt als die „Fünf Ps“ (people, planet, prosperity, peace, partnership). Die Präambel verspricht auch, „auf dieser gemeinsamen Reise, die wir heute antreten, niemanden zurückzulassen““.11 Dieser Leitspruch, „niemanden zurückzulassen“ (Leave No One Behind), findet sodann Ausdruck in dem Universalitätsanspruch, den die Politische Erklärung in ihrem Abs. 5 formuliert:

„Diese Agenda ist von beispielloser Reichweite und Bedeutung. Sie wird von allen Ländern akzeptiert und ist auf alle anwendbar...Ibre Ziele und Zielvorgaben sind universell." 42

Dieser universelle Anspruch ist ein echtes Novum der Agenda 2030, gerade im Vergleich zu den vorangegangenen Millenniumsentwicklungszielen, und war von Beginn an tragender Bestandteil der Verhandlungen. ${ }^{43} \mathrm{Wa}$ ren die Millenniumsentwicklungsziele eine klare Entwicklungsagenda, die auf einen Nord-Süd-Transfer ausgerichtet waren, sind die Nachhaltigen Entwicklungsziele an alle Mitgliedstaaten gerichtet: Alle Staaten, inklusive der Industriestaaten, sind aufgefordert, nationale Umsetzungsstrategien zu entwickeln und diese im Rahmen des Hochrangigen Politischen Forums vorzulegen (dieser Prozess ist prinzipiell freiwillig, es ist aber mit politi-

39 UN Dok. A/Res/70/1, Anhang.

40 UN Dok. A/69/313, Anhang; UN Dok. A/Res/71/313, Anhang.

41 UN Dok. A/Res/70/1, Anhang, Präambel.

42 UN Dok. A/Res/70/1, Ziff. 5.

43 Dodds/Donoghue/Leiva Roesch, Negotiating the Sustainable Development Goals (Fn. 18), S. 34. 
schen Kosten verbunden, sich nicht dem freiwilligen Review-Verfahren zu unterziehen). ${ }^{44}$ Der Universalitätsanspruch richtet sich auch auf die inhaltliche Breite der Agenda: Sie zeichnet sich gerade dadurch aus, dass sie beansprucht, ökologische Nachhaltigkeit mit sozialen und wirtschaftlichen Aspekten zusammenzudenken.

Während die Agenda 2030 formal eine nicht rechtsverbindliche Empfehlung der Generalversammlung darstellt, zeugt schon der ausführliche und hochrangig besetzte Verhandlungsprozess unter Einbeziehung verschiedenster Akteure von der hohen politischen Relevanz, die diesem Dokument beigemessen wurde und wird. Die Agenda 2030 und ihre Indikatoren beziehen sich zudem an verschiedenen Stellen auf bereits bestehende Rahmenwerke und eine ganze Reihe völkerrechtlich verbindlicher Instrumente, und sie formulieren konkrete Zielvorgaben für die innerstaatliche Rechtsordnung. Nachfolgend seien einige Beispiele genannt:

- Ziel 1 zur Armutsbekämpfung legt in seinem Unterziel 4 fest, dass alle Menschen gleiche Rechte auf wirtschaftliche Ressourcen sowie Zugang zu Grundeigentum haben sollen; gemessen wird der Anteil der erwachsenen Bevölkerung, der sichere Landnutzungs- und Besitzrechte hat;

- Ziel 5 zur Geschlechtergleichstellung zielt auf die Beendigung der Diskriminierung der Frau, hier wird u.a. gemessen, welche gesetzlichen Rahmenbedingungen hierfür bestehen. Darüber hinaus wird auch die Zahl der Gesetze über den Zugang zu sexueller und reproduktiver Gesundheit und reproduktiven Rechten gemessen (Unterziel 5.6);

- Ziel 10 zur Beseitigung von Ungleichheiten nicht nur zwischen, sondern auch in Ländern verweist in seinen Indikatoren global auf internationale Menschenrechtsstandards und Antidiskriminierungsrecht; ${ }^{45}$

- Ziel 13 zum Klimawandel verweist explizit auf die Klimarahmenkonvention der Vereinten Nationen;

- Ziel 14 zu Ozeanen und Meeren verweist explizit auf das Seerechtsübereinkommen der Vereinten Nationen.

An zahlreichen Stellen wird außerdem deutlich, dass die Ziele nur dann erreicht werden können, wenn auf der innerstaatlichen Ebene Änderungen herbeigeführt werden, die in aller Regel mit der Verabschiedung oder Reform nationaler Gesetze einhergehen. Dazu zählen zahlreiche Ziele und

44 Vgl. Fukuda-Parr, Sustainable Development Goals (Fn. 30), S. 768.

45 Zum Zusammenhang von Menschenrechten und den Nachhaltigen Entwicklungszielen s. a. Birkenkötter, Zum Verhältnis der 2030 Agenda für Nachhaltige Entwicklung und der internationalen Menschenrechte, zfmr 2021, 26. 
Unterziele im sozialen Bereich, etwa zum Gesundheitswesen oder der Bildung, aber auch im Bereich der Infrastruktur oder der Städteplanung. Gekoppelt mit den Verweisen auf geltende, völkerrechtliche Verträge und ihrer hohen politischen Signalkraft ist die Agenda 2030 also weit mehr als eine einfache Empfehlung der VN-Generalversammlung an ihre Mitgliedstaaten, sondern ein Instrument, das auf nationale Politik- und Rechtsveränderungen zielt.

Dazu kommt, dass die Agenda, wie bereits erwähnt, die wohl derzeit wichtigste Leitschnur für das System der Vereinten Nationen mit seinen zahlreichen Unterorganisationen darstellt. Das gilt insbesondere für das Geflecht entwicklungspolitischer Programme, Fonds und Entitäten. Diese haben nicht nur ihre eigene Arbeit an den 17 Zielen ausgerichtet, sondern werben auch beständig für ihre Umsetzung auf staatlicher und lokaler Ebene. Über die Hälfte der Bediensteten der Vereinten Nationen arbeiten heute nicht mehr im Hauptquartier oder den anderen Standorten der Vereinten Nationen, sondern auf lokaler Ebene. Das VN-Entwicklungsprogramm UNDP unterhält in über 140 der 193 Mitgliedstaaten der Vereinten Nationen Länderbüros; diese wirken darauf hin, dass politische Aktionsprogramme - und hier zuvörderst die Agenda 2030 - auch national umgesetzt werden. Ein wichtiger Bestandteil der Arbeit der VN-Länderbüros ist die Unterstützung nationaler Entwicklungspläne; hier wirken die Vereinten Nationen durch ihr operatives Geschäft im Feld darauf hin, dass die Agenda 2030 mit ihren Zielen angemessen in den nationalen Entwicklungsstrategien aufgegriffen wird. Bis zu einem gewissen Grad spielen hier auch Konditionalitäten internationaler Finanzinstitutionen eine Rolle: Internationale Finanzinstitutionen knüpfen Kredite häufig an die Umsetzung bestimmter internationaler Normen, und diese werden u.a. durch VN-Entitäten, mit denen internationale Finanzinstitutionen vor Ort zusammenarbeiten, geprägt. ${ }^{46}$

Dieser letzte Aspekt ist natürlich nicht für alle Länder gleichermaßen relevant: Die 50 Mitgliedstaaten der Vereinten Nationen, in denen die Vereinten Nationen kein Länderbüro unterhalten, sind durchweg industrialisierte Länder. An der institutionellen Präsenz der Vereinten Nationen vor Ort hat sich auch durch den Universalitätsanspruch der Agenda 2030 nichts geändert. Hier zeigt sich also, dass zwar der Anspruch der Agenda 2030 für alle Länder gleich formuliert ist, seine Umsetzung aber von den jeweiligen Umständen eines Landes und auch von der institutionellen Prä-

46 Zur Auswirkung solcher Konditionalitäten sogar auf verfassunggebende Prozesse siehe Sripati, Constitution-Making under UN Auspices, 2020. 
senz der Vereinten Nationen vor Ort abhängen kann, wobei in Deutschland der Prozess zur Deutschen Nachhaltigkeitsstrategie im Kanzlerinnenamt und damit politisch hochrangig aufgehängt ist. ${ }^{47}$

Die Agenda 2030 richtet sich außerdem auch direkt an nicht-staatliche und sub-staatliche Akteure. Bereits in der Präambel heißt es: „Alle Länder und alle Interessenträger werden diesen Plan in kooperativer Partnerschaft umsetzen." Mit dem Ziel 11 ist ein eigenes Ziel für Städte ausgerufen; lokale Regierungen werden hier unmittelbar zum Handeln aufgefordert. ${ }^{48}$ Und schließlich spricht das Ziel 17 von einer Globalen Partnerschaft für Nachhaltige Entwicklung, die durch Multi-Akteur-Partnerschaften und öffentlich-private Partnerschaften unterstützt werden soll. Die Agenda 2030 formuliert Zielvorgaben für Bereiche wie dem Arbeits- und Sozialwesen, dem Gesundheitswesen, der Gleichstellungspolitik, dem Infrastrukturund Planungsrecht, Energie, Umweltpolitik oder Verwaltungsstrukturen, also für solche Themenbereiche, die lange als domaine réservé staatlicher Souveränität galten. Die Agenda wirkt also keinesfalls als horizontales Koordinationsrecht zwischen Staaten, sondern sie formuliert den Anspruch, in den Staat hineinzuwirken. Dieser Anspruch wird aber nicht als ein klares, linear-vertikales Einwirken verstanden.

\section{Zugang zur Rechtssetzung am Beispiel der Agenda 2030: Legitimatorische Herausforderungen für das zeitgenössische Völkerrecht}

In der Agenda 2030 geht es nicht um eine hierarchisierte „top-down“ Relation, sondern um die nicht-lineare Vernetzung verschiedener Akteursebenen. Im Folgenden wird auf Möglichkeiten der begrifflichen Erfassung dieses Phänomens eingegangen, sowie darauf, inwieweit sich hieraus legitimatorische Probleme jedenfalls für demokratisch verfasste Staaten ergeben, und ob diese durch einen Zugang nicht-staatlicher Akteure zum Rechtssetzungsprozess aufgefangen werden können. Dies soll erneut am Beispiel der Agenda 2030 und konkret dem Zugang von Frauen zum Verhandlungsprozess untersucht werden.

47 Bundesregierung, Nachhaltigkeitsstrategie neu aufgelegt, https://www.bundesregi erung.de/breg-de/themen/nachhaltigkeitspolitik/nachhaltigkeitsstrategie-2021-187 3560 (letzter Aufruf am 2. April 2021).

$48 \mathrm{Zu}$ Sustainable Development Goal 11 siehe die Beiträge in Aust/Du Plessis (Hrsg.), The Globalisation of Urban Governance, 2018. 
1. Rekursivität als zentrales Merkmal: Transnationale Rechtsordnungen und experimentelles Regieren jenseits des Staates

Das zeitgenössische Völkerrecht zeichnet sich - das zeigt sich an dem bis hierher Beschriebenen - durch seine Verhandlung in institutionalisierten Prozessen, die ihrerseits wiederum starke Pfadabhängigkeiten aufweisen, aus. Es ist seiner Natur nach prozesshaft, und nicht statisch. Halliday und Shaffer haben mit dem Begriff der "transnationalen Rechtsordnungen“ bzw. des "transnationalen rechtlichen Ordnens" einen Versuch vorgelegt, Entwicklungen, wie sie sich an der Agenda 2030 zeigen, begrifflich zu erfassen und zu theoretisieren. ${ }^{49}$ Sie definieren transnationale Rechtsordnungen als solche normativen Ordnungen, die im Rahmen einer transnational, also über staatliche Grenzen hinweg agierenden Institution geschaffen werden, formell oder informell, direkt oder indirekt in mehrere staatliche Rechtsordnungen hineinwirken, und die in eine Form gegossen werden, die als Recht erkennbar ist, wobei das Formerfordernis nicht im Sinne der traditionellen Quellenlehre, sondern weiter, nämlich als jede formalisierte Verschriftlichung, verstanden wird. So entsteht ein prozessuales Verständnis transnationaler Rechtsordnungen. ${ }^{50}$

Natürlich ist das von Halliday und Shaffer vorgelegte Konzept transnationalen rechtlichen Ordnens nur eine mögliche Deutung des Begriffs des Transnationalen, ein Begriff, der in den letzten Jahren erheblich an Prominenz gewonnen und damit zugleich vielleicht an Schärfe verloren hat. ${ }^{51}$ Philipp Jessup prägte den Begriff „transnational law“ bereits 1956 und verstand darunter jedes Recht, das einen grenzüberschreitenden Sachverhalt regelt, also sowohl klassisches Völkerrecht als auch internationales Privatrecht, sowie eine Kategorie sog. „anderer Regeln“, die in keine der beiden vorgenannten Kategorien passen. ${ }^{52}$ Schon bei Jessup bleibt diese letzte Kategorie vage und undefiniert, ${ }^{53}$ und die Konzeptionalisierung von Halliday und Shaffer leidet vielleicht am meisten darunter, dass nicht ganz klar ist, was eine als Recht erkennbare Form ist, außer, dass wohl schriftliche Do-

49 Halliday/Shaffer, Transnational Legal Orders, 2015. Im Original klingt das transnationale rechtliche Ordnen mit transnational legal ordering/transnational legal orders etwas geschmeidiger, vgl. S. 4.

50 Halliday/Shaffer, Transnational Legal Orders (Fn. 49), S. 7-21.

51 Für eine Übersicht über verschiedene Bedeutungen und Konzeptionalisierungen des Begriffs der „transnationalen Rechtsordnung“ siehe Halliday, Theorizing Transnational Legal Orders, Annu.Rev.LawSoc.Sci 2016, 231 (233-246).

52 Jessup, Transnational Law, 1956, S. 2.

53 Halliday (Fn. 51), S. 232. 
kumente gemeint sind - wobei hier wohl Gewohnheitsrecht gerade auch als völkerrechtliche Kategorie zu kurz kommt und gerade im Kontext der Vereinten Nationen durchaus ein Unterschied besteht, ob ein bestimmtes Thema etwa im Rahmen einer unverbindlichen Erklärung der Generalversammlung verabschiedet wird, oder in der Form eines völkerrechtlichen Vertrags.

Gleichwohl ist Hallidays und Shaffers Begriffsbildung für das Verständnis eines Instruments wie der Agenda 2030 hilfreich, und zwar vor allem, weil ihre Theorie transnationaler Rechtsordnungen die Dichotomie zwischen nationalem und internationalem Recht durchbricht. Zugleich heben Halliday und Shaffer das Element der Rekursivität hervor, welches durch das prozessuale Verständnis noch verstärkt wird: Verschiedene transnationale Rechtsordnungen interagieren in mehrfachen, rekursiven Beziehungen miteinander, aber auch mit nationalen Rechtsordnungen. Damit wird die Wirkung einzelner Instrumente immer erst durch ihre Interaktion mit anderen Ordnungen verständlich. Zwei klassische Trennungen werden hier aufgehoben: die Trennung zwischen internationaler und nationaler Rechtssphäre einerseits und die Trennung zwischen Rechtssetzung und Rechtsumsetzung andererseits. ${ }^{54}$

Der Begriff der Rekursivität erlaubt in der Durchbrechung der Trennung zwischen internationaler und nationaler Rechtssphäre, dass gleichzeitige, vielfältige, rekursive Beziehungen zwischen verschiedenen transnationalen und nationalen Rechtsordnungen zu einem offenen, iterativen Lernprozess führen, der im Mehrebenensystem zwischen den verschiedenen Ebenen oszilliert. Diesen experimentellen Charakter hat zuletzt Gráinne de Búrca für ein besseres Verständnis der Wirkungsweise internationaler Menschenrechte fruchtbar gemacht. ${ }^{55}$ Auch sie betont das Prozesshafte zeitgenössischer völkerrechtlicher Instrumente und stellt eine Theorie transnationalen experimentellen Regierens vor. ${ }^{56}$ Diese Theorie geht davon aus, dass das Zusammenwirken fünf notwendiger Faktoren - grundsätzliche Übereinstimmung, dass ein gemeinschaftliches Problem besteht, Einigung auf grobe Zielvorgaben zu seiner Lösung auf globaler Ebene, Einbindung lokaler Akteure in die Umsetzung der Zielvorgaben und dadurch Anpassung an lokale Gegebenheiten, Feedback der lokalen Akteure an die globale Ebene, und regelmäßige Überprüfung und Anpassung des

54 Halliday/Shaffer, Transnational Legal Orders (Fn. 49), S. 37-42.

55 De Búrca, Human Rights Experimentalism, AJIL 111 (2017), 277; De Búrca, Reframing Human Rights in a Turbulent Era, 2021.

56 Dazu grundlegend De Búrca/Keohane/Sabel, Global Experimentalist Governance, B.J.Pol.S. 44 (2014), 477 (477-478). 
globalen Rahmens - zu einer normativ wünschenswerten deliberativen und partizipativen Form globalen Regierens führt. ${ }^{57}$

De Búrca unterstreicht, dass eine Theorie experimentellen transnationalen Regierens, deren zentrales Merkmal gerade in der Rekursivität besteht, auf die auch Halliday/Shaffer verweisen, deswegen normativ attraktiv ist, weil sie besonders die Aspekte angemessener Teilhabe an transnationalen Prozessen sowie dem Lernen von der lokalen Ebene hervorhebt. ${ }^{58}$ Gerade solche Akteure, die durch globale Normen besonders angesprochen werden oder von einer bestimmten Problematik besonders betroffen sind, ${ }^{59}$ sollen im Rahmen experimentellen globalen Regierens an der Formung transnationaler Rechtsordnungen beteiligt und damit der partizipative Aspekt gestärkt werden. Damit wird eine große Leerstelle transnationaler Rechtsordnungen in den Blick genommen, nämlich die Frage nach der Rechenschaftspflicht rechtsetzender Akteure gegenüber denen, die von den Normen betroffen sind. Jessup hatte das transnationale Recht vor allem als problemlösungsorientierte Kategorie entwickelt; über die Frage der Beteiligung und dadurch resultierende, bessere Qualität des Rechts, aber auch dessen Akzeptanz, hatte er sich wenige Gedanken gemacht. ${ }^{60}$

\section{Zugang zur Rechtssetzung bei der Agenda 2030: Das Beispiel der Women's Major Group}

Vor diesem Hintergrund soll nun der Zugang zur Rechtssetzung im Rahmen der Verhandlungen zur Agenda 2030 näher in den Blick genommen werden, und zwar am Beispiel der Women's Major Group, die als eine der einflussreichsten Major Groups in diesem Verhandlungsprozess gilt. ${ }^{61}$ Die Women's Major Group hatte zwei Ziele: Die Agenda 2030 sollte ein eigenständiges Ziel zur Geschlechtergleichstellung und Geschlechtergerechtigkeit enthalten, und Geschlechtergerechtigkeit sollte außerdem

57 De Búrca (Fn. 55), S. 282; De Búrca/Keohane/Sabel (Fn. 56), S. 478.

58 De Búrca (Fn. 55), 281.

59 Siehe zur Frage der Betroffenheit als Kriterium für Beteiligungsmöglichkeiten Hasl, Das Konzept der Betroffenheitskollektive im Völkerrecht: Zugangsschlüssel für vulnerable Personengruppen zur internationalen Rechtserzeugung?, in diesem Band.

60 Michaels, in: Zumbansen (Hrsg.), The Many Lives of Transnational Law, 2020, S. 441 (449-450).

61 Esquivel, Power and the Sustainable Development Goals: a feminist analysis, Gender\&Development 24 (2016), 18. 
als Querschnittsthema in allen anderen Zielen verankert werden. ${ }^{62}$ Die Anstrengungen der Women's Major Group waren außerordentlich erfolgreich: Mit Ziel 5 existiert ein eigenständiges Ziel der Geschlechtergleichstellung, und ein Bericht der VN-Entität für Frauen (UN Women) hat ausführlich dargelegt, dass sämtliche 17 Ziele eine Geschlechterdimension enthalten und diese auch sehr häufig in den Unterzielen und Indikatoren selbst enthalten ist. ${ }^{63}$

Welche Organisationen waren in der Women's Major Group vertreten, welche hatten Zugang zu den Verhandlungsprozessen? Hier muss trotz des erklärten Ziels der Women's Major Group, einen Schwerpunkt auf Führung durch Frauen aus dem Globalen Süden zu legen, ${ }^{64}$ ein differenziertes Bild verschachtelter Konditionalitäten gezeichnet werden. Die Women's Major Group hat einen ausgeprägten Fokus auf transparente Beteiligungsstrukturen, durch die vor allem Expertise aus dem Globalen Süden in die Vereinten Nationen getragen werden soll. Während der Verhandlungen der Agenda 2030, insbesondere während der für die Zivilgesellschaft wohl wichtigsten frühen Phase der Offenen Arbeitsgruppe, ${ }^{65}$ verfügte die Women's Major Group über ein selbstorganisiertes System mit Vertreter*innen in allen Weltregionen. ${ }^{66}$ Gleichwohl waren vermutlich mangelnde Ressourcen ein Hauptgrund dafür, dass nur eine Vertreterin von außerhalb des amerikanischen Kontinents regelmäßig zu den Verhandlungen der Offenen Arbeitsgruppe nach New York reisen konnte. Das Tagesgeschäft vor Ort wurde in großen Teilen von amerikanischen Organisationen übernommen. ${ }^{67}$

Es zeigt sich an dieser Stelle vor allem ein strukturelles Problem der Major Groups: Wie schon die weiter oben erwähnte Liste der verschiedenen Major Groups zeigt, sind die einzelnen Gruppen sehr unterschiedlich. Manche Major Groups vertreten bestimmte Berufsgruppen (Arbeiter*innen, Landwirtschaft), andere bestimmte Bevölkerungsgruppen (Frauen, Kinder und Jugend, Ältere), wieder andere sind Akteure der öffentlichen

62 International Women's Health Coalition, Power Lessons: Advocacy and the 2030 Agenda, 2017, S. 11-12.

63 UN Women, Turning Promises Into Action, 2018, Kapitel 3.

64 Gabizon, Women's movements' engagement in the SDGs: lessons learned from the Women's Major Group, Gender\&Development 24 (2016), 99 (104).

65 Dodds/Donoghue/Leiva Roesch, Negotiating the Sustainable Development Goals (Fn. 18), S. 62.

66 Gabizon, Lessons learned (Fn. 64), 104-105.

67 Siehe die Tabelle mit globalen und regionalen Partnerorganisationen bei Gabizon, Lessons learned (Fn. 64), 106. 
Hand (lokale Regierungen). Eine Vertretung von Städten und Gemeinden wird anders organisiert sein und auch andere Ziele verfolgen als eine Vertretung indigener Bevölkerungsgruppen oder eine Vertretung der Wirtschaft. Die Gruppen sind auch in sich nicht homogen, höchst unterschiedlich organisiert und diffus vernetzt. Einzelne Aktivist*innen sind häufig sowohl in ihrer persönlichen Position als auch stellvertretend für eine oder mehrere Organisationen und in einem oder mehreren zivilgesellschaftlichen Bündnissen vertreten. ${ }^{68}$ Dass die Women's Major Group vor allem während der Phase der Offenen Arbeitsgruppe weitestgehend einheitlich agierte, erklärt vermutlich einen Teil ihres Erfolgs. ${ }^{69}$

Obwohl die Women's Major Group einen so starken Fokus auf die Beteiligung von Organisationen aus dem Globalen Süden legte, ist sie nicht frei von Machtgefällen, die insbesondere mit Ressourcen verbunden sind, die Beteiligung erst ermöglichen. Die finanzielle Dimension wurde bereits erwähnt. Besonders solvente und damit wirkmächtige Organisationen sind, selbst wenn sie aus Ländern des Globalen Südens stammen, in der Regel in ihrer Finanzierung von Programmen der Entwicklungsministerien industrialisierter Staaten abhängig, die wiederum häufig an bestimmte Konditionen geknüpft sind. Darüber hinaus sind solvente Organisationen häufig mehrfach vertreten: Viele Regierungen etwa europäischer, aber auch amerikanischer Staaten haben im Bereich der Gleichstellungspolitik Frauen aus der Zivilgesellschaft in ihre Regierungsdelegationen aufgenommen. Das ist insbesondere für Delegationen zur jährlichen Frauenrechtskonferenz der Fall, deren Verhandlungen und Themen - als Follow-up-Prozess zur Vierten Weltfrauenkonferenz in Peking - auch auf die Offene Arbeitsgruppe und die Verhandlung des Nachhaltigkeitsziels zur Geschlechtergleichstellung ausstrahlten. Auf individueller Ebene wird das durch Teilnahme an Regierungsdelegationen erworbene Wissen geteilt und breit gestreut; gleichwohl verbleiben durch Ressourcenunterschiede hervorgerufene Wissens-, Zugangs- und damit Machtgefälle auf der strukturellen Ebene bestehen.

Ein Erklärungsansatz für diese Schieflage liegt möglicherweise in der Entstehungsgeschichte der Major Groups, die, wie erwähnt, 1992 im Rahmen des ersten Rio-Gipfels ins Leben gerufen wurden. In der Agenda 21 werden die Major Groups nicht als primäre Akteure der Rechtsetzung betrachtet, sondern als besonders relevante gesellschaftliche Gruppen, die in der Umsetzung nachhaltiger Entwicklung besonders berücksichtigt wer-

68 Gabizon, Lessons learned (Fn. 64), 103.

69 International Women's Health Coalition (Fn. 62), S. 21. 
den sollten. ${ }^{70}$ Die Major Groups wurden also nicht mit Blick auf Zugang zur Völkerrechtssetzung geschaffen, dies ergab sich vielmehr in den darauffolgenden Jahren aus den Aktivitäten der Major Groups heraus. Das erklärt auch, warum einige Major Groups deutlich aktiver sind als andere. Heute hat sich, das hat der Verhandlungsprozess der Agenda 2030 gezeigt, das System der Major Groups als Beteiligungsmodus im Bereich der Nachhaltigen Entwicklung in den Vereinten Nationen durchgesetzt. Andere Teile des VN-Systems, etwa das Welternährungsprogramm, zeigen, dass andere Beteiligungsmodi und dahinterstehende Logiken denkbar sind. ${ }^{71}$

\section{Transnationale Rechtsordnungen und Herausforderungen ibrer demokratischen Legitimation}

Bis hierhin ist argumentiert worden, dass sich das zeitgenössische Völkerrecht, exemplifiziert durch die Agenda 2030, durch Prozesshaftigkeit sowie nicht-lineare und heterarchische Vernetzungen verschiedener Akteursund damit auch verschiedener Rechtsebenen im Mehrebenensystem auszeichnet. Das vorgestellte Modell der transnationalen Rechtsordnungen versucht, dies begrifflich zu erfassen und stellt dabei auch gerade auf die Einwirkung transnationaler Rechtsordnungen in nationale Rechtsordnungen ab. Auch die Theorie experimentellen transnationalen Regierens legt ein besonderes Augenmerk auf die Interaktion transnationaler und lokaler Akteure und Rechtsebenen. Mit dem Anspruch transnationaler Rechtsordnungen, auf nationale Rechtsordnungen auszustrahlen, und der damit einhergehenden Auflösung einer klaren Trennung zwischen Innen und Außen stellt sich jedenfalls für demokratisch verfasste Staaten die Frage nach der demokratischen Legitimation völkerrechtlicher Instrumente. ${ }^{72}$

Traditionell wird diese Frage da gestellt, wo internationale Organisationen unmittelbare Herrschaftsgewalt ausüben, also etwa da, wo sie im Rahmen einer Übergangsverwaltung hoheitliche Befugnisse erlangt haben, oder wo Rechtsakte internationaler Akteure sich unmittelbar auf Individuen auswirken, etwa im Bereich gezielter Sanktionen des VN-Sicherheitsrats. $^{73}$ Dabei wird letztlich nach funktionalen Äquivalenten staatlicher Hoheitsgewalt im Völkerrecht gesucht, die allerdings nur selten gefunden

70 Vgl. Agenda 21, UN Dok. A/CONF.121/56 (Vol. III), Abschnitt III.

71 Vgl. Hasl (Fn. 59).

72 Ley, Opposition im Völkerrecht, 2015, Kapitel 2 und 4.

73 Krieger, Die Herrschaft der Fremden, AöR 133 (2008), 315, insb. 328-338. 
werden - außerhalb der zwei prominenten Fälle, nämlich dem Kosovo und Ost-Timor, haben die Vereinten Nationen nie wieder im gleichen Umfang uneingeschränkte, staatsgleiche, unmittelbare Hoheitsgewalt im Rahmen einer Übergangsverwaltung ausgeübt. Stattdessen erhalten sie in der Regel beratende Unterstützungsmandate, auf dessen Grundlage sie dann die Regierungen fragiler Staaten während eines Übergangsprozesses anleiten, wie etwa zuletzt das Mandat der Mission UNITAMS im Sudan. ${ }^{74}$ Die Auswirkungen einer solchen Unterstützung auf etwa einen nationalen, verfassungsgebenden Prozess sollten gleichwohl nicht unterschätzt werden. ${ }^{75}$

Eine Problembeschreibung, die ausschließlich auf unmittelbare Ausübung von Hoheitsgewalt abstellt, geht also in Zeiten der Diffusion von Hoheitsgewalt im Mehrebenensystem an einer Vielzahl von Konstellationen, die die heutige transnationale Rechtsordnung prägen, vorbei. Sie verkennt, an welcher Stelle sich das Demokratieproblem im Völkerrecht eigentlich primär stellt. Vielmehr ist auch die vermittelte Einwirkung transnationaler Rechtsordnungen in den Blick zu nehmen. Jürgen Habermas hat beobachtet, dass die Konstitutionalisierung des Völkerrechts gerade nicht die „logische Fortsetzung der konstitutionellen Zähmung einer naturwüchsig operierenden Staatsgewalt ${ }^{\text {"76 }}$ ist, sondern dass die Konstitutionalisierung des Völkerrechts in eine zur Genealogie des Verfassungsstaats gegenläufige Richtung erfolgt: Dem klassischen Völkerrecht ging es um den Ausgleich von Machtasymmetrien, nicht um die Begründung von Hoheitsgewalt wie im Verfassungsstaat. Dann aber ist bereits die Suche nach funktionalen Äquivalenten staatlicher Hoheitsgewalt durch internationale Organisationen der falsche Anknüpfungspunkt für die Frage nach der demokratischen Legitimation völkerrechtlicher Normen. Stattdessen setzt Habermas auf zweierlei: einerseits auf die Zunahme demokratisch verfasster Staaten, die an der Völkerrechtssetzung teilnehmen, ${ }^{77}$ andererseits auf die Internationalisierung demokratischer Rechtsprinzipien, die aus de-

74 Vgl. UN Dok. S/Res/2524 (2020), Ziff. 2.

75 Dazu Sripati, Constitution-Making (Fn. 46), Kapitel 6, die eine Übersicht über alle verfassungsunterstützenden Maßnahmen der Vereinten Nationen in rund 40 Ländern bereitstellt.

76 Habermas, Hat die Konstitutionalisierung des Völkerrechts noch eine Chance? [2004], in: ders., Philosophische Texte Band 4: Politische Theorie (Studienausgabe 2009), S. 331.

77 Ähnlich Möllers, Demokratie - Zumutungen und Versprechen, 2008, S. 83: „Die einfachste Form einer demokratischen Welt bestünde in der Demokratisierung aller Staaten." 
mokratischen Lernprozessen hervorgegangen sind. ${ }^{78}$ Beides fließt in der heutigen Völkerrechtsordnung da zusammen, wo es eine internationale Norm demokratischer Regierungsformen gibt (wenngleich es sich eher nicht um ein subjektives Recht auf demokratische Herrschaft handelt und auch nicht handeln sollte), ${ }^{79}$ die von den Vereinten Nationen im Rahmen ihres operativen Geschäfts gerade in Postkonflikt-Staaten, aber auch in solchen Staaten, in denen die Vereinten Nationen mit ihren Entwicklungsprogrammen vor Ort präsent sind, beworben und gefördert wird. ${ }^{80}$ Gleichwohl wäre dann aber jede völkerrechtliche Norm, an deren Setzung auch nicht demokratisch verfasste Staaten teilhaben, selbst nie demokratisch legitimiert. ${ }^{81}$

In Zeiten transnationaler Rechtsordnungen ist aber dieses Problem möglicherweise dadurch entschärft, dass internationale Rechtsnormen erst durch die rekursiven Beziehungen zwischen unterschiedlichen Akteuren ihr eigentliches Potenzial entfalten. Das soll nicht heißen, dass internationale Normen transnationaler Rechtsordnungen keine Auswirkungen auf die innerstaatliche Rechtsordnung haben, im Gegenteil. Aber der Geltungsanspruch ist kein linearer und unmittelbarer, sondern stets durch Interaktion vermittelt. Dann muss im demokratischen Verfassungsstaat danach gefragt werden, ob bestehende verfassungsrechtliche Mechanismen ausreichen, um eine demokratische Legitimation der zunehmend nach innen, aber gleichzeitig auch nur vermittelt wirkenden transnationalen Rechtsordnungen zu gewährleisten - jedenfalls dann, wenn weder eine Parlamentarisierung auf der internationalen rechtsetzenden Ebene ${ }^{82}$ noch eine Demokratisierung aller an der Völkerrechtsordnung teilhabenden Staaten zu erwarten ist. Die deutsche Verfassungsdogmatik knüpft bei der Frage nach der demokratischen Legitimation auswärtigen Handelns vor allem an das Zustimmungserfordernis nach Art. 59 Abs. 2 GG für völkerrechtliche Verträge an. Art. 59 Abs. 2 gilt zunächst einmal auch für solche

78 Habermas, Konstitutionalisierung (Fn. 76), S. 341-342.

79 So aber Franck, The emerging right to democratic governance, AJIL 86 (1992), 46; wie hier mit unterschiedlichen Argumenten Boysen, Remnants of a Constitutional Moment, und Besson, The Human Right to Democracy in International Law, beide in von Arnauld/von der Decken/Susi (Hrsg.), Cambridge Handbook of New Human Rights, 2020, S. 465-480 und S. 481-489.

80 Zur Begriffsgeschichte der Demokratie im System der Vereinten Nationen Haack, The United Nations democracy agenda, 2011.

81 Möllers, Demokratie (Fn. 77), S. 83.

82 Zum Problemkomplex der internationalen Parlamentarisierung siehe Ley, Opposition (Fn. 72), Kapitel 12; dies., Zur Politisierung des Völkerrechts, AVR 50 (2012), 191 (202-215). 
völkerrechtlichen Verträge, durch die internationale Organisationen errichtet werden, soweit diesen keine Hoheitsgewalt übertragen wird, was jedenfalls vom Bundesverfassungsgericht im Sinne einer unmittelbaren Durchgriffsgewalt verstanden wird. ${ }^{83}$ Für Systeme kollektiver Sicherheit, zu denen auch die Vereinten Nationen gezählt werden, und deren Rechtsakte, soll sich die demokratische Legitimation aus Art. 24 Abs. 2 iVm Art. 59 Abs. 2 GG ergeben, wobei die einmal erteilte Zustimmung auch bei Funktionsänderungen der internationalen Organisation greifen soll, solange diese keine Vertragsänderung erfordert, also intra vires verbleibt. ${ }^{84}$

Ob diese Konstruktion da begründet ist, wo internationale Organisationen heute wesentlich neue und zum Zeitpunkt des Beitritts der Bundesrepublik zu dieser Organisation auch nicht vorhersehbare Aufgaben übernommen haben und Recht in neuartiger Weise erzeugt wird, kann an dieser Stelle nicht abschließend behandelt werden. Skepsis ist hier jedenfalls angebracht, ${ }^{85}$ gerade weil das Kriterium der unmittelbaren Durchgriffskraft auf nur wenige völkerrechtliche Normkonstellationen zutreffen dürfte. Das Problem ließe sich allerdings mindestens praktisch entschärfen, wenn internationale Rechtssetzungsprozesse, wie etwa der Verhandlungsprozess der Agenda 2030, stärker an die nationale Legislative rückgebunden wären und durch diese begleitet würden, etwa im Wege regelmäßiger parlamentarischer Unterrichtung, aber auch möglicherweise durch die Einbindung von Repräsentant*innen verschiedener Parlamentsfraktionen in internationale Verhandlungsprozesse. ${ }^{86}$

Schließlich kann auch die gleiche Teilhabe betroffener Akteure die demokratische Legitimation transnationaler Rechtsordnungen dann stärken, wenn außerhalb der hierarchisch organisierten Legitimationsketten weitere Partizipationsmöglichkeiten geschaffen werden, also die Möglichkeit verschiedener, parallel verlaufender demokratischer Legitimationsstränge

83 Vgl. BVerfG, Nichtannahmebeschluss vom 27. Januar 2010, - 2 BvR 2253/06 -, Rn. 14.

84 So BVerfGE 104, 151 - NATO-Konzept. Zu unterschiedlichen Logiken in der Legitimation auswärtiger Gewalt je nach Teilbereich jetzt Ley, Zwischen parlamentarischer Routine und exekutiven Kernbereichen: Eine Rekonstruktion der Kompetenzverteilung der auswärtigen Gewalt von Parlament und Regierung unter dem Grundgesetz, AöR (2021; im Erscheinen).

85 So auch Ley (Fn. 84).

86 Eine Teilnahme von Parlamentarier*innen an Regierungsdelegationen gab es etwa im Rahmen der WTO-Verhandlungskonferenz in Seattle, vgl. Ley, Opposition (Fn. 72), S. 248-250. 
genutzt wird. ${ }^{87}$ Aus praktischer Sicht ist das kein einfaches Unterfangen, weil, wie am Beispiel der Women's Major Group exemplarisch dargestellt, Beteiligungsmöglichkeiten derzeit sehr ungleich verteilt sind. Gleichwohl wird hier vertreten, dass die gleichzeitige Stärkung parlamentarischer Begleitung internationaler Prozesse einerseits sowie die Schaffung gleicher Beteiligungsmöglichkeiten für verschiedene Interessengruppen unmittelbar an internationalen Verhandlungsprozessen andererseits die derzeit beste Möglichkeit bietet, die demokratische Legitimation transnationaler Rechtsordnungen zu stärken. Vor fast dreißig Jahren schrieb Brun-Otto Bryde: Der „Aufbau transnationaler demokratischer Institutionen ist eine Aufgabe, für die es keine Blaupause gibt und die ein erhebliches Maß an Kreativität verlangt“ ${ }^{88}$ Daran hat sich fast dreißig Jahre später wenig geändert. Die hiesigen Ausführungen verstehen sich als ein erster Schritt, überhaupt erst einmal das Problem angemessen zu beschreiben.

87 Grundlegend Bryde, Die bundesrepublikanische Volksdemokratie als Irrweg der Demokratietheorie, Staatswissenschaft und Staatspraxis 5 (1994), 305.

88 Bryde, Volksdemokratie als Irrweg (Fn. 87), 308. 
